\title{
DETERMINAÇÃO DO VALOR DE REFERÊNCIA DO ALUGUEL DE IMÓVEIS RESIDENCIAIS EMPREGANDO O MÉTODO TODIM
}

\author{
Luís Alberto Duncan Rangel* \\ Escola de Eng. Ind. Metalúrgica de Volta Redonda \\ Universidade Federal Fluminense (UFF) \\ Volta Redonda - RJ \\ duncan@metal.eeimvr.uff.br
}

\author{
Luiz Flávio Autran Monteiro Gomes \\ Ibmec/RJ \\ Rio de Janeiro - RJ \\ autran@,ibmecrj.br
}

* Corresponding author / autor para quem as correspondências devem ser encaminhadas

Recebido em 12/2006; aceito em 04/2007

Received December 2006; accepted April 2007

\begin{abstract}
Resumo
As avaliações de alternativas em presença de múltiplos critérios, quantitativos e qualitativos, são realizadas nas mais diversas áreas. Este artigo apresenta um estudo dessa natureza, conduzido junto às imobiliárias da cidade de Volta Redonda, RJ, visando auxiliar a definição do valor de referência de aluguel de imóveis residenciais. Para isto, faz-se uso do método TODIM, do Apoio Multicritério à Decisão. Com a finalidade de obter os valores de referência o mais próximo possível dos valores de mercado, alguns imóveis que tinham acabado de ser alugados foram inseridos na pesquisa, conhecendo-se desta forma o seu valor de aluguel. Assim, ao empregar o Método TODIM para realizar a ordenação dos imóveis com diferentes características, em função dos critérios empregados na análise e de suas importâncias relativas para os decisores, obtém-se a ordenação de todos os imóveis, e, como conseqüência disto, chega-se às diversas faixas de valor de aluguel dos imóveis em estudo. Uma análise de sensibilidade dos resultados numéricos obtidos complementa o estudo.
\end{abstract}

Palavras-chave: método TODIM; apoio multicritério à decisão; mercado imobiliário.

\begin{abstract}
Setting rent reference values for residential housing units is normally performed under multiple, conflicting criteria. This article shows how the TODIM method of Multicriteria Decision Aiding can be of a significant help in that task. A case study was carried through in the city of Volta Redonda, in the State of Rio de Janeiro, Brazil. The TODIM method allows considering quantitative as well as qualitative evaluation criteria and is founded on Prospect Theory. In order to obtain a maximum adherence of rent reference values to market values, some housing units that had just been rent were taken into account in the study. The application of the TODIM method produced the ordering of all such housing units and then led to identifying ranges for rent. A sensitivity analysis complemented the study by providing useful insights on the numerical results that were obtained.
\end{abstract}

Keywords: TODIM method; multicriteria decision aiding; real estate market. 


\section{Introdução}

Este artigo aborda a utilização de um método do Apoio Multicritério à Decisão na avaliação de imóveis residenciais que estão disponíveis para alugar na cidade de Volta Redonda, no Estado do Rio de Janeiro. Na maioria das vezes os critérios empregados para fazer a avaliação das alternativas são conflitantes. Por exemplo, o que seria mais valorizado: uma casa pequena, antiga, em um excelente bairro residencial, próximo ao centro; ou uma residência grande, nova, com piscina e área de lazer, num bairro distante do centro da cidade?

Existem diversos métodos empregados para fazer a avaliação de um imóvel para aluguel (Guimarães Neto, 1992; Pessôa, 1992). Dentre estes métodos, os dois mais empregados no mercado imobiliário são:

- Método Comparativo: São verificados imóveis semelhantes em localidades próximas a do imóvel em questão e, a partir daí, comparativamente, é definido o valor de mercado para o aluguel. Este método é o mais praticado no ramo imobiliário e normalmente é o que apresenta o valor mais próximo do valor de mercado;

- Método de Renda: Define o valor do aluguel como sendo uma porcentagem do valor de venda do imóvel. É simples de se aplicar, mas dependendo da oferta e procura na região em questão, não conduz a valores competitivos e praticáveis no mercado.

O método TODIM (Gomes, 2007; Gomes, Araya \& Carignano, 2004), do Apoio Multicritério à Decisão, é capaz de ordenar as alternativas de acordo com as preferências dos decisores. Para isso, incorpora em sua formulação a Teoria dos Prospectos (Kahneman \& Tversky, 1979) que considera a aversão e a propensão ao risco durante o processo decisório. Neste estudo, o método TODIM foi empregado para ordenar alternativas de imóveis, que foram selecionados dentre os disponíveis para alugar, na cidade de Volta Redonda, no Estado do Rio de Janeiro. Após esta ordenação, haverá maior facilidade para definir os valores de aluguel, uma vez que foram inseridos no conjunto de alternativas alguns imóveis anteriormente avaliados, isto é, com seus valores de aluguel já definidos.

O presente artigo foi elaborado da forma explicada a seguir. Após a introdução, nas subseções 2.1 e 2.2, respectivamente, apresenta-se uma breve introdução ao Apoio Multicritério à Decisão e à Teoria dos Prospectos. Em seguida, na subseção 2.3 apresenta-se a teoria do método TODIM. O estudo de caso realizado em si está contido na seção 3 do artigo.

\section{Quadro de Referência Teórico}

\subsection{O Apoio Multicritério à Decisão}

O Apoio Multicritério à Decisão é o campo da Pesquisa Operacional empregado quando se deseja realizar seleção, classificação, ordenação, ou descrição de alternativas passíveis de solucionarem um problema de decisão, na presença simultânea de critérios quantitativos e qualitativos (Roy \& Bouyssou, 1993; Keeney \& Raiffa, 1993; Belton \& Stewart, 2002; Gomes, Gomes \& Almeida, 2006). Desta forma, sob estas considerações, defronta-se tal campo com uma ampla gama de problemas de decisão. Assim, diversos métodos analíticos têm sido propostos e empregados na prática, como os métodos ELECTRE - ELECTRE I (Roy, 1968), ELECTRE II (Roy \& Bertier, 1973), ELECTRE III (Roy, 1978), ELECTRE IV (Roy \& Hugonnard, 1982), ELECTRE IS (Roy \& Skalka, 1984) -; os métodos 
PROMÉTHÉE (Brans, Mareschal \& Vincke, 1984; Brans \& Mareschal, 1990); o método AHP (Saaty, 1990); o método UTA (Jacquet-Lagrèze \& Siskos, 1982); o método UTAModificado (Rangel, 2002); o método TODIM (Gomes, 2007) e o método MACBETH (Bana e Costa \& Vansnick, 1997), dentre outros, bem como algumas variações adicionais destes métodos. Cada um desses métodos analíticos em geral possui características e axiomas específicos para se resolver um problema de decisão. No estudo relatado no presente artigo, que tem como objetivo a ordenação de alternativas - isto é, resolver problemas do tipo $\mathrm{P} \gamma$ (Roy \& Bouyssou, 1993) -, empregou-se o método TODIM, que tem a característica, importante para o problema em pauta, de modelar explicitamente os padrões de preferência dos decisores em presença de risco. Para tanto, o método TODIM fundamenta-se na Teoria dos Prospectos, desenvolvida originalmente por Daniel Kahneman e Amos Tversky na década de 70 (Kahneman \& Tversky, 1979).

\subsection{A Teoria dos Prospectos}

Esta teoria foi desenvolvida a partir das pesquisas de dois psicólogos israelenses, Kahneman \& Tversky (1979) apud Gomes, Araya \& Carignano (2004). O objetivo dessas pesquisas era avaliar o comportamento humano durante a tomada de decisões em situações de risco. Estes psicólogos observaram que, nas situações que envolvem ganhos, há a tendência do ser humano em ser mais conservador em relação ao risco, ou seja, as pessoas preferem optar por um ganho menor, porém seguro, a correr um risco para obter um ganho maior. Por outro lado, em situações que envolvem perdas, as pessoas mostram-se mais propensas ao risco, isto é, elas preferem correr o risco de ter perdas maiores (caso exista a possibilidade de não perderem nada) a aceitar uma perda menor, porém segura.

A Teoria dos Prospectos utiliza a função de valor para explicar a aversão e a propensão ao risco. Esta função tem a forma de "S", representada na Figura 1. Acima do eixo horizontal, considerado como referência desta análise, tem-se uma curva côncava representando os ganhos, e, abaixo do eixo horizontal, tem-se uma curva convexa representando as perdas. A parte côncava reflete a aversão ao risco em face aos ganhos, e a parte convexa, por sua vez, simboliza a propensão ao risco, quando se trata de perdas.

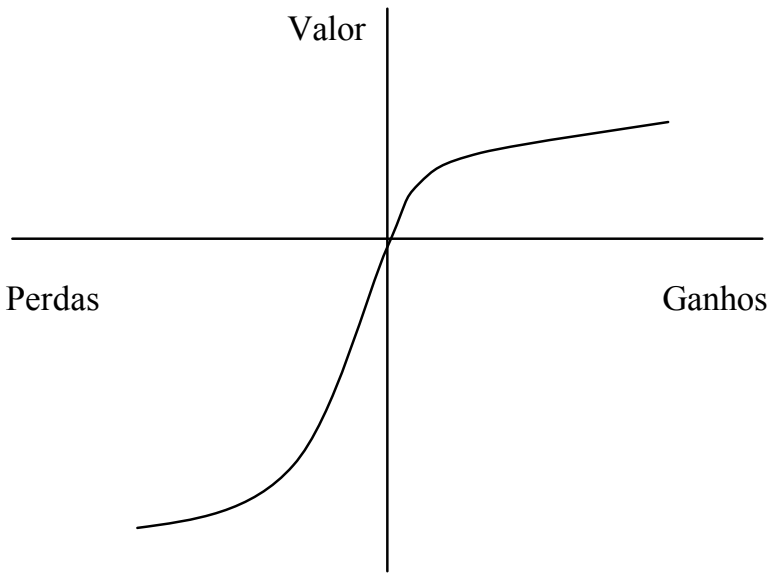

Figura 1 - Função de Valor do Método TODIM (Gomes \& Lima, 1992a). 


\subsection{O Método TODIM}

O método multicritério TODIM (acrônimo de TOmada de Decisão Interativa e Multicritério), concebido em sua forma atual ao início dos anos 90 (Gomes, 2007), é provavelmente o único método multicritério fundamentado na Teoria dos Prospectos. Isto significa que existe definitivamente uma teoria subjacente ao método - e uma teoria tão forte que foi mesmo objeto de Prêmio Nobel de Economia concedido em 2002 (Roux, 2002). Assim sendo, enquanto praticamente todos os demais métodos multicritério partem da premissa de que $o$ tomador de decisão decide buscando sempre a solução correspondente ao máximo de alguma medida global de valor (por exemplo, o maior valor possível de uma função de utilidade multiatributo - no caso da MAUT), o método TODIM faz uso da noção de uma medida global de valor calculável pela aplicação do paradigma em que consiste a Teoria dos Prospectos. Com isto, o método se alicerça sobre uma descrição - já sobejamente comprovada por evidências empíricas - de como as pessoas efetivamente decidem em face ao risco.

No entanto, de modo a poder aplicar esse paradigma a uma base de dados proveniente de cálculos e de juízos de valor, o método TODIM deve testar formas específicas das funções de perdas e de ganhos. Estas, uma vez validadas empiricamente, servirão para construir-se a função de diferença aditiva do método, a qual fornece medidas de dominância de cada alternativa sobre cada outra alternativa. Embora pareça complicado ter-se que testar aquela validade da aplicação do paradigma à base de dados - o que poderia eventualmente obrigar o analista de decisão a usar outras formas das funções de perdas e de ganhos -, na verdade não o é, pois, desde os primeiros usos práticos do método TODIM, ainda no início dos anos noventa do século passado, as duas mesmas formas matemáticas tem sido empregadas com sucesso (ou seja, tem sido validadas empiricamente).

A partir da construção da já mencionada função de diferença aditiva do TODIM - a qual funciona como uma função de valor multiatributo e, como tal, deve ter seu uso também validado, pela verificação da condição de independência preferencial mútua (Keeney \& Raiffa, 1993; Clemen \& Reilly, 2001) - o método conduz a uma ordenação global das alternativas. Observe-se que se constrói a função de valor multiatributo - ou função de diferença aditiva - do método TODIM partindo-se de uma projeção das diferenças entre os valores de duas alternativas quaisquer (percebida em relação a cada critério) sobre um critério referencial ou critério de referência.

O método TODIM faz uso de comparações por pares entre os critérios de decisão, possuindo recursos tecnicamente simples e corretos para eliminar eventuais inconsistências provenientes dessas comparações. Também permite efetuar-se juízos de valor em uma escala verbal, utilizar uma hierarquia de critérios, juízos de valor nebulosos e fazer uso de relações de interdependência entre alternativas.

Embora o TODIM tenha parte de suas raízes na tese de doutorado do segundo autor deste artigo (Gomes, 1976), sua atual formulação, fundamentada na Teoria dos Prospectos e adquirida no início da década de 90, inspirou-se em Salminen (1992). Este autor, por sua vez, foi um dos primeiros pesquisadores a associarem, do ponto de vista computacional, a Teoria dos Prospectos ao Apoio Multicritério à Decisão, embora sem a aparente preocupação em desenvolver um método analítico de ampla aplicabilidade (Gomes, 2007).

Em Roy \& Bouyssou (1993), sobre o método TODIM, afirmam que este é: 
“... um método tendo como base a Escola Francesa e a Escola Americana. Combina aspectos provenientes da Teoria de Utilidade Multiatributo, do método AHP e dos métodos ELECTRE” (p. 638), (Gomes, 2007).

A idéia, presente na formulação do método TODIM, de introduzir-se na mesma função de valor multiatributo expressões de perdas e de ganhos aufere a esse método alguma similaridade com os métodos PROMÉTHÉE, que fazem uso da mencionada noção de fluxo líquido de superação. Barba-Romero \& Pomerol (1997) compreenderam isto, notando que:

“... baseia-se em uma noção bastante parecida com a de fluxo líquido, no sentido do PROMÉTHÉE” (p. 229).

O capítulo 5 de Gomes, Araya \& Carignano (2004) explica com detalhes o método TODIM. Encontram-se algumas das várias aplicações já realizadas deste método em: Gomes \& Lima (1992a; 1992b); Gomes \& Duarte (1998); Gomes, Duarte \& Moraes (1999); Trotta, Nobre \& Gomes (1999); Costa, Almeida \& Gomes (2003); e Passos \& Gomes (2005). Duas outras aplicações foram divulgadas recentemente (Mourão \& Gomes, 2006; Rangel, Silva \& Silvério, 2006).

Considere-se um conjunto de $n$ alternativas a serem ordenadas na presença de $m$ critérios quantitativos ou qualitativos. Para determinar os pesos dos critérios, o método TODIM emprega uma matriz de comparação por pares entre critérios. Para formar esta matriz, faz-se uso da escala de Saaty (1990). Como, no entanto, toda matriz de comparações por pares pode conter alguma inconsistência, decorrente da violação da transitividade, o método TODIM possui um recurso que, ao mesmo tempo que respeita os juízos de valor que conduziram à essa matriz inicial, corrige-os de uma forma sistemática, eliminando por completo a eventual inconsistência. Uma vez assim constituída a matriz corrigida de comparações por pares entre os critérios, a aplicação do método TODIM prossegue na direção da obtenção dos pesos dos critérios, usando-se para isto as seguintes duas etapas: (i) somam-se os valores ao longo de cada coluna dessa matriz corrigida; (ii) calculam-se os recíprocos dessas somas; (iii) dividese cada um desses recíprocos pelas soma dos recíprocos: os valores desta forma obtidos serão os pesos dos critérios. Após a definição desses pesos dos critérios, pede-se a especialistas que estimem, para cada um dos critérios qualitativos $c$, a contribuição de cada alternativa i para o objetivo associado ao critério. Este método requer que os valores das avaliações, dos critérios em relação às alternativas, sejam numéricos e que sejam normalizados, portanto os critérios qualitativos avaliados numa escala verbal são transformados numa escala cardinal. As avaliações dos critérios quantitativos são obtidas através dos desempenhos das alternativas em relação aos critérios, como por exemplo, nível de ruído em decibéis, potência de um motor em HP, nota de um aluno em uma disciplina etc (Gomes, Araya \& Carignano, 2004).

Após a avaliação das alternativas em relação a todos os critérios, obtém-se a matriz de avaliação, onde os valores são todos numéricos. Faz-se então a normalização dos mesmos, empregando-se, por exemplo, para determinado critério, a divisão do valor de uma alternativa pela soma dos valores de todas as alternativas. Essa normalização é feita para cada critério, obtendo-se assim uma matriz, onde todos os valores estão entre zero e um, denominada matriz de desejabilidades parciais $\mathrm{W}=\left[\mathrm{W}_{\mathrm{nm}}\right]$, conforme se mostra na Tabela 1 (Gomes, Araya \& Carignano, 2004). 
Tabela 1 - Matriz de Desejabilidades Parciais.

\begin{tabular}{|c|c|c|c|c|c|c|}
\hline \multirow{2}{*}{ Alternativas } & \multicolumn{5}{|c|}{ Critérios } \\
\cline { 2 - 7 } & $\mathrm{C}_{1}$ & $\mathrm{C}_{2}$ & $\ldots$ & $\mathrm{C}_{\mathrm{j}}$ & $\ldots$ & $\mathrm{C}_{\mathrm{m}}$ \\
\hline $\mathrm{A}_{1}$ & $\mathrm{~W}_{11}$ & $\mathrm{~W}_{12}$ & $\ldots$ & $\mathrm{W}_{1 \mathrm{j}}$ & $\ldots$ & $\mathrm{W}_{1 \mathrm{~m}}$ \\
\hline $\mathrm{A}_{2}$ & $\mathrm{~W}_{21}$ & $\mathrm{~W}_{22}$ & $\ldots$ & $\mathrm{W}_{2 \mathrm{j}}$ & $\ldots$ & $\mathrm{W}_{2 \mathrm{~m}}$ \\
\hline$\ldots$ & $\ldots$ & $\ldots$ & $\ldots$ & $\ldots$ & $\ldots$ & \\
\hline $\mathrm{A}_{\mathrm{i}}$ & $\mathrm{W}_{\mathrm{i} 1}$ & $\mathrm{~W}_{\mathrm{i} 2}$ & $\ldots$ & $\mathrm{W}_{\mathrm{ij}}$ & $\ldots$ & $\mathrm{W}_{\mathrm{im}}$ \\
\hline$\ldots$ & $\ldots$ & $\ldots$ & $\ldots$ & & $\ldots$ & $\ldots$ \\
\hline $\mathrm{A}_{\mathrm{n}}$ & $\mathrm{W}_{\mathrm{n} 1}$ & $\mathrm{~W}_{\mathrm{n} 2}$ & $\ldots$ & $\mathrm{W}_{\mathrm{nj}}$ & $\ldots$ & $\mathrm{W}_{\mathrm{nm}}$ \\
\hline
\end{tabular}

Após a atribuição dos pesos dos critérios e de sua normalização, necessita-se calcular as matrizes de dominância parciais e a matriz de dominância final. Admita-se agora que um dos $m$ critérios pode ser considerado como sendo um critério de referência r; este poderá ser, por exemplo, identificado como sendo o critério de maior peso. Assim, $\mathrm{a}_{\mathrm{rc}}$ representa a taxa de substituição do critério em análise c em relação ao critério de referência $r$. A medida de dominância de cada alternativa i sobre cada alternativa $\mathrm{j}$, incorporada agora à Teoria dos Prospectos, é dada pela expressão matemática (1):

$$
\delta(i, j)=\sum_{c=1}^{m} \Phi(i, j), \forall(i, j)
$$

onde:

$$
\Phi(i, j)= \begin{cases}\sqrt{\frac{a_{r c}\left(W_{i c}-W_{j c}\right)}{\sum_{c=1}^{m} a_{r c}}} & \text { se }\left(W_{i c}-W_{j c}\right)>0, \\ 0 & \text { se }\left(W_{i c}-W_{j c}\right)=0, \\ \frac{-1}{\theta} \sqrt{\frac{\left(\sum_{c=1}^{m} a_{r c}\right)\left(W_{j c}-W_{i c}\right)}{a_{r c}}} & \text { se }\left(W_{i c}-W_{j c}\right)<0,\end{cases}
$$

assim:

- $\delta(i, j)$ representa a medida de dominância da alternativa i sobre a alternativa j;

- m é o número de critérios;

- c é um critério qualquer, para $\mathrm{c}=1, \ldots, \mathrm{m}$;

- $a_{\mathrm{rc}}$ é a taxa de substituição do critério c pelo critério de referência r;

- $\mathrm{W}_{\mathrm{ic}}$ e $\mathrm{W}_{\mathrm{jc}}$ são, respectivamente, os pesos das alternativas i e j em relação a c;

- $\theta$ é o fator de atenuação das perdas.

O fator $\Phi_{c}(\mathrm{i}, \mathrm{j})$ representa a parcela de contribuição do critério c à função $\delta(i, j)$, quando se compara a alternativa $\mathrm{i}$ com a alternativa $\mathrm{j}$. Caso o valor de $\mathrm{W}_{\mathrm{ic}}-\mathrm{W}_{\mathrm{jc}}$ seja positivo, representará um ganho para a função $\delta(i, j)$ e, portanto, será usada a expressão de $\Phi_{c}(i, j)$ correspondente, isto é, à equação (2). Caso $\mathrm{W}_{\mathrm{ic}}-\mathrm{W}_{\mathrm{jc}}$ seja nulo, será atribuído o valor nulo para $\Phi_{c}(\mathrm{i}, \mathrm{j})$, isto é, a equação $(3)$, e se $\mathrm{W}_{\mathrm{ic}}-\mathrm{W}_{\mathrm{jc}}$ for negativo, $\Phi_{\mathrm{c}}(\mathrm{i}, \mathrm{j})$ será representado pela equação (4). 
Depois de calculadas as diversas matrizes de dominância parciais, uma para cada critério, obtém-se a matriz de dominância final $\delta(\mathrm{i}, \mathrm{j})$, através da soma dos elementos das diversas matrizes.

A matriz de dominância final é então normalizada, usando-se a expressão (5), para obter o valor global de cada alternativa. Deve-se interpretar cada número calculado como a medida da desejabilidade ou utilidade global, ou, simplesmente, como o valor de uma alternativa específica. A ordenação das alternativas origina-se da ordenação de seus respectivos valores.

$$
\xi_{i}=\frac{\sum_{j=1}^{n} \delta(i, j)-\min \sum_{j=1}^{n} \delta(i, j)}{\max \sum_{j=1}^{n} \delta(i, j)-\min \sum_{j=1}^{n} \delta(i, j)}
$$

Portanto, o método TODIM determina, a partir das preferências expressas por um decisor ou um grupo de decisores, uma escolha, ao ordenar todas as alternativas. Mudando-se tal conjunto de preferências, pode-se eventualmente chegar a um novo resultado, através de uma análise de sensibilidade, como se comenta mais adiante na seção 3.5.

\section{Estudo de Caso}

A cidade de Volta Redonda está situada ao sul do estado do Rio de Janeiro. Tem aproximadamente 256.000 habitantes e possui atualmente um grande número de imóveis, tanto residenciais como comerciais, alugados ou à disposição para aluguel.

Esta pesquisa busca determinar um valor de referência para o aluguel dos imóveis residenciais que estão para serem alugados, servindo desta maneira como uma importante fonte de informação para os proprietários e para as imobiliárias.

Para realizar a avaliação de imóveis foi necessário identificar quais critérios deveriam ser considerados neste estudo. Para tanto, foi elaborada uma pesquisa junto a diversas imobiliárias da cidade de Volta Redonda e foram feitas entrevistas com especialistas deste ramo, que trabalham em avaliação de imóveis.

\subsection{Definição dos Critérios}

A partir dos pontos de vistas explicitados nas entrevistas com profissionais do ramo imobiliário, identificaram-se os critérios mais relevantes para esta análise, descritos a seguir:

$\mathrm{C}_{1}$ - Localização:

É o mais importante critério de avaliação dos imóveis para aluguel. É um critério qualitativo que busca definir se o imóvel está ou não em uma localização bem valorizada. Para avaliar o desempenho das alternativas em relação a este critério, definiu-se a escala apresentada na Tabela 2. Desta forma, um imóvel com "boa localização", nesta avaliação, recebe uma pontuação igual a 4 . 
Tabela 2 - Valoração do Critério $C_{1}$ - Localização.

\begin{tabular}{|c|c|}
\hline Localização & Pontuação Associada \\
\hline Periferia & 1 \\
\hline Periferia / Média localização & 2 \\
\hline Média localização & 3 \\
\hline Boa localização & 4 \\
\hline Excelente localização & 5 \\
\hline
\end{tabular}

$\mathrm{C}_{2}$ - Área de Construção:

Critério quantitativo que retrata o tamanho da construção do imóvel. Neste estudo, a unidade de medida utilizada foi o metro quadrado $\left(\mathrm{m}^{2}\right)$.

$\mathrm{C}_{3}$ - Qualidade da Construção:

Critério qualitativo que determina o padrão de acabamento do imóvel. Foram definidos três níveis de acabamento e suas respectivas pontuações, que são apresentados na Tabela 3. Considerando uma residência que tenha sido construída com um "alto padrão de acabamento", este imóvel recebe a pontuação igual a 3, valor máximo para este critério de avaliação.

Tabela 3 - Valoração do Critério $\mathrm{C}_{3}$ - Qualidade da Construção.

\begin{tabular}{|c|c|}
\hline Qualidade da Construção & Pontuação Associada \\
\hline Baixo padrão de acabamento & 1 \\
\hline Médio padrão de acabamento & 2 \\
\hline Alto padrão de acabamento & 3 \\
\hline
\end{tabular}

$\mathrm{C}_{4}$ - Estado de Conservação:

Critério qualitativo utilizado para avaliar o estado geral do imóvel. Este critério considera se houve ou não reforma do imóvel, e também suas condições de moradia. A Tabela 4, a seguir, apresenta a pontuação associada ao estado de conservação do imóvel. Por exemplo, um apartamento em condições de moradia, necessitando de pequenos reparos, ou seja, com "médio estado de conservação", recebe a pontuação igual a 2. Este critério não está diretamente relacionado ao critério $\mathrm{C} 3$, pois o estado de conservação independe do padrão de acabamento do imóvel. Um imóvel pode ter um alto padrão de acabamento, com material de grande qualidade, mas estar precisando de reformas, devido à sua idade.

Tabela 4 - Valoração do Critério $\mathrm{C}_{4}$ - Estado de Conservação.

\begin{tabular}{|c|c|}
\hline Estado de Conservação & Pontuação Associada \\
\hline Ruim & 1 \\
\hline Médio & 2 \\
\hline Bom & 3 \\
\hline Ótimo & 4 \\
\hline
\end{tabular}


$\mathrm{C}_{5}$ - Número de Vagas na Garagem:

Critério quantitativo e muito valorizado por pessoas que necessitam alugar um imóvel e possuem um ou mais veículos.

$\mathrm{C}_{6}$ - Número de Cômodos:

Critério quantitativo que considera para a valoração a quantidade dos seguintes cômodos existentes no imóvel: salas, quartos, copa/cozinha e banheiros. Destaca-se aqui a diferença entre este critério e o critério $\mathrm{C}_{2}$, que retrata a área de construção. É interessante avaliar o imóvel também pelo número de cômodos, pois podem estar em questão dois imóveis com o mesmo tamanho total, mas um deles pode ter poucos cômodos grandes, e o outro, com maior número de cômodos menores.

$\mathrm{C}_{7}$ - Atrativos:

Critério qualitativo que traduz a existência de dependências de lazer, como piscina e churrasqueira, além de outros. É avaliado de acordo com a Tabela 5. Uma casa que tenha piscina e churrasqueira recebe 4 pontos.

Tabela 5 - Valoração do Critério $\mathrm{C}_{7}$ - Atrativos.

\begin{tabular}{|c|c|}
\hline Atrativos & Pontuação Associada \\
\hline Sem atrativos & 0 \\
\hline Quintal ou terraço & 1 \\
\hline Churrasqueira & 2 \\
\hline Piscina & 3 \\
\hline Piscina, churrasqueira ou outros & 4 \\
\hline
\end{tabular}

$\mathrm{C}_{8}$ - Segurança:

Critério qualitativo empregado para retratar as condições de segurança do imóvel. A Tabela 6 apresenta a avaliação do imóvel segundo este critério. Por exemplo, um apartamento que tenha porteiro e câmeras de segurança recebe pontuação igual a 1.

Tabela 6 - Valoração do Critério $\mathrm{C}_{8}$ - Segurança.

\begin{tabular}{|c|c|}
\hline Segurança & Pontuação Associada \\
\hline Não existe nenhuma segurança adicional & 0 \\
\hline $\begin{array}{c}\text { Existência de porteiros e câmeras de segurança em apartamento. } \\
\text { Casas com guaritas de segurança nas ruas. }\end{array}$ & 1 \\
\hline
\end{tabular}

Todos os critérios acima definidos são critérios de maximização, ou seja, quanto maior for a pontuação obtida na avaliação das alternativas em relação a cada critério, melhor será o seu desempenho.

\subsection{Pesos dos Critérios}

De acordo com a importância dos critérios empregados nesta pesquisa para avaliar os imóveis, seus respectivos pesos foram definidos pelos decisores através da valoração direta, e posteriormente normalizados. Ambas as informações são apresentadas na Tabela 7. 
Tabela 7 - Pesos dos Critérios.

\begin{tabular}{|c|c|c|c|}
\hline Critério & Descrição & Peso Atribuído & Peso Normalizado \\
\hline $\mathrm{C}_{1}$ & Localização & 5 & 0,25 \\
\hline $\mathrm{C}_{2}$ & Área construída & 3 & 0,15 \\
\hline $\mathrm{C}_{3}$ & Qualidade da construção & 2 & 0,10 \\
\hline $\mathrm{C}_{4}$ & Estado de conservação & 4 & 0,20 \\
\hline $\mathrm{C}_{5}$ & Número de vagas na garagem & 1 & 0,05 \\
\hline $\mathrm{C}_{6}$ & Número de cômodos & 2 & 0,10 \\
\hline $\mathrm{C}_{7}$ & Atrativos & 1 & 0,05 \\
\hline $\mathrm{C}_{8}$ & Segurança & 2 & 0,10 \\
\hline
\end{tabular}

\subsection{As Alternativas do Processo de Decisão}

Neste estudo de caso foram avaliados quinze imóveis localizados em diferentes bairros da cidade de Volta Redonda. Os imóveis e suas respectivas localidades não foram identificados, por julgar-se desnecessária a sua exposição neste artigo. Apenas definiram-se as características dos imóveis e estes foram designados por $A_{1}, A_{2}, A_{3}, \ldots, A_{15}$. A seguir descrevem-se os quinze imóveis empregados na avaliação. São eles:

- $\mathrm{A}_{1}$ - Casa em localização média, com $290 \mathrm{~m}^{2}$ de área construída, alto padrão de acabamento, bom estado de conservação, com uma vaga na garagem, 6 cômodos, piscina, churrasqueira e outros atrativos, não apresenta sistema de segurança.

- $\mathrm{A}_{2}$ - Casa em boa localização, com $180 \mathrm{~m}^{2}$ de área construída, médio padrão de acabamento, estado de conservação médio, com uma vaga na garagem, 4 cômodos, quintal e terraço, não apresenta sistema de segurança.

- $\mathrm{A}_{3}$ - Casa em localização média, com $347 \mathrm{~m}^{2}$ de área construída, baixo padrão de acabamento, estado de conservação médio, com duas vagas na garagem, 5 cômodos, quintal amplo, não apresenta sistema de segurança.

- $\mathrm{A}_{4}$ - Casa em localização média, com $124 \mathrm{~m}^{2}$ de área construída, médio padrão de acabamento, bom estado de conservação, com duas vagas na garagem, 5 cômodos, pomar com árvores frutíferas, piscina e churrasqueira, não apresenta sistema de segurança.

- $\mathrm{A}_{5}$ - Casa em excelente localização, com $360 \mathrm{~m}^{2}$ de área construída, alto padrão de acabamento, ótimo estado de conservação, com quatro vagas na garagem, 9 cômodos, quintal e guaritas de segurança nas ruas do bairro.

- $\mathrm{A}_{6}$ - Casa localizada entre a periferia e o centro da cidade (periferia/média localização), com $89 \mathrm{~m}^{2}$ de área construída, médio padrão de acabamento, bom estado de conservação, com uma vaga na garagem, 5 cômodos, quintal, não apresenta sistema de segurança.

- $\quad \mathrm{A}_{7}$ - Apartamento localizado na periferia, com $85 \mathrm{~m}^{2}$ de área construída, baixo padrão de acabamento, estado de conservação ruim, com uma vaga na garagem, 4 cômodos, portaria com segurança.

- $\mathrm{A}_{8}$ - Apartamento em excelente localização, com $80 \mathrm{~m}^{2}$ de área construída, médio padrão de acabamento, bom estado de conservação, com uma vaga na garagem, 6 cômodos, portaria com segurança. 
- $\mathrm{A}_{9}$ - Apartamento localizado entre a periferia e o centro da cidade (periferia/média localização), com $121 \mathrm{~m}^{2}$ de área construída, médio padrão de acabamento, bom estado de conservação, sem garagem, 6 cômodos, não apresenta sistema de segurança.

- $\mathrm{A}_{10}$ - Casa localizada entre a periferia e o centro da cidade (periferia/média localização), com $120 \mathrm{~m}^{2}$ de área construída, baixo padrão de acabamento, bom estado de conservação, com uma vaga na garagem, 5 cômodos, quintal amplo, não apresenta sistema de segurança.

- $\mathrm{A}_{11}$ - Casa com boa localização, com $280 \mathrm{~m}^{2}$ de área construída, médio padrão de acabamento, apresentando um estado de conservação médio, com duas vagas na garagem, 7 cômodos, possuindo ainda sistema de segurança.

- $\mathrm{A}_{12}$ - Apartamento localizado na periferia, com $90 \mathrm{~m}^{2}$ de área construída, baixo padrão de acabamento, estado de conservação ruim, uma vaga na garagem, 5 cômodos, não possui segurança adicional.

- $\mathrm{A}_{13}$ - Apartamento localizado na periferia com média localização, com $160 \mathrm{~m}^{2}$ de área construída, com alto padrão de acabamento, em bom estado de conservação, com 2 vagas na garagem, 6 cômodos, apresentando segurança adicional.

- $\mathrm{A}_{14}$ - Apartamento com média localização, com $320 \mathrm{~m}^{2}$ de área construída, apresenta alto padrão de acabamento, está em bom estado de conservação, com 2 vagas na garagem, 8 cômodos, possuindo ainda sistema adicional de segurança.

- $\mathrm{A}_{15}$ - Casa localizada com boa localização, com $180 \mathrm{~m}^{2}$ de área construída, com padrão médio de acabamento, em ótimo estado de conservação, com uma vaga na garagem, 6 cômodos, possuindo ainda sistema de segurança adicional.

A Tabela 8, contendo o Desempenho das Alternativas, apresenta de forma completa a avaliação dos imóveis presentes na análise em relação aos critérios selecionados pelos decisores.

Tabela 8 - Desempenho das Alternativas.

\begin{tabular}{|c|c|c|c|c|c|c|c|c|}
\hline \multirow{2}{*}{ Alternativas } & \multicolumn{9}{|c|}{ Critérios } \\
\cline { 2 - 10 } & $\mathrm{C}_{1}$ & $\mathrm{C}_{2}$ & $\mathrm{C}_{3}$ & $\mathrm{C}_{4}$ & $\mathrm{C}_{5}$ & $\mathrm{C}_{6}$ & $\mathrm{C}_{7}$ & $\mathrm{C}_{8}$ \\
\hline $\mathrm{A}_{1}$ & 3 & 290 & 3 & 3 & 1 & 6 & 4 & 0 \\
\hline $\mathrm{A}_{2}$ & 4 & 180 & 2 & 2 & 1 & 4 & 2 & 0 \\
\hline $\mathrm{A}_{3}$ & 3 & 347 & 1 & 2 & 2 & 5 & 1 & 0 \\
\hline $\mathrm{A}_{4}$ & 3 & 124 & 2 & 3 & 2 & 5 & 4 & 0 \\
\hline $\mathrm{A}_{5}$ & 5 & 360 & 3 & 4 & 4 & 9 & 1 & 1 \\
\hline $\mathrm{A}_{6}$ & 2 & 89 & 2 & 3 & 1 & 5 & 1 & 0 \\
\hline $\mathrm{A}_{7}$ & 1 & 85 & 1 & 1 & 1 & 4 & 0 & 1 \\
\hline $\mathrm{A}_{8}$ & 5 & 80 & 2 & 3 & 1 & 6 & 0 & 1 \\
\hline $\mathrm{A}_{9}$ & 2 & 121 & 2 & 3 & 0 & 6 & 0 & 0 \\
\hline $\mathrm{A}_{10}$ & 2 & 120 & 1 & 3 & 1 & 5 & 1 & 0 \\
\hline $\mathrm{A}_{11}$ & 4 & 280 & 2 & 2 & 2 & 7 & 3 & 1 \\
\hline $\mathrm{A}_{12}$ & 1 & 90 & 1 & 1 & 1 & 5 & 2 & 0 \\
\hline $\mathrm{A}_{13}$ & 2 & 160 & 3 & 3 & 2 & 6 & 1 & 1 \\
\hline $\mathrm{A}_{14}$ & 3 & 320 & 3 & 3 & 2 & 8 & 2 & 1 \\
\hline $\mathrm{A}_{15}$ & 4 & 180 & 2 & 4 & 1 & 6 & 1 & 1 \\
\hline
\end{tabular}


No estudo de caso, o fator de atenuação de perdas $\theta$ tem valor igual a 1 , o que significa que as perdas contribuirão com seu valor real para o valor global. Para a implementação do método, é necessário que estes desempenhos sejam normalizados. A matriz de desempenho normalizada é chamada matriz de desejabilidades parciais, apresentada na Tabela 9 abaixo.

Tabela 9 - Matriz de Desejabilidades Parciais.

\begin{tabular}{|c|c|c|c|c|c|c|c|c|}
\hline \multirow{2}{*}{ Alternativas } & \multicolumn{9}{|c|}{ Critérios } \\
\cline { 2 - 9 } & $\mathrm{C}_{1}$ & $\mathrm{C}_{2}$ & $\mathrm{C}_{3}$ & $\mathrm{C}_{4}$ & $\mathrm{C}_{5}$ & $\mathrm{C}_{6}$ & $\mathrm{C}_{7}$ & $\mathrm{C}_{8}$ \\
\hline $\mathrm{A}_{1}$ & 0,068 & 0,103 & 0,1 & 0,075 & 0,045 & 0,069 & 0,174 & 0 \\
\hline $\mathrm{A}_{2}$ & 0,091 & 0,064 & 0,067 & 0,05 & 0,045 & 0,046 & 0,087 & 0 \\
\hline $\mathrm{A}_{3}$ & 0,068 & 0,123 & 0,033 & 0,05 & 0,091 & 0,057 & 0,043 & 0 \\
\hline $\mathrm{A}_{4}$ & 0,068 & 0,044 & 0,067 & 0,075 & 0,091 & 0,057 & 0,174 & 0 \\
\hline $\mathrm{A}_{5}$ & 0,114 & 0,127 & 0,1 & 0,1 & 0,182 & 0,103 & 0,043 & 0,143 \\
\hline $\mathrm{A}_{6}$ & 0,045 & 0,031 & 0,067 & 0,075 & 0,045 & 0,057 & 0,043 & 0 \\
\hline $\mathrm{A}_{7}$ & 0,023 & 0,03 & 0,033 & 0,025 & 0,045 & 0,046 & 0 & 0,143 \\
\hline $\mathrm{A}_{8}$ & 0,114 & 0,028 & 0,067 & 0,075 & 0,045 & 0,069 & 0 & 0,143 \\
\hline $\mathrm{A}_{9}$ & 0,045 & 0,043 & 0,067 & 0,075 & 0 & 0,069 & 0 & 0 \\
\hline $\mathrm{A}_{10}$ & 0,045 & 0,042 & 0,033 & 0,075 & 0,045 & 0,057 & 0,043 & 0 \\
\hline $\mathrm{A}_{11}$ & 0,091 & 0,099 & 0,067 & 0,05 & 0,091 & 0,08 & 0,13 & 0,143 \\
\hline $\mathrm{A}_{12}$ & 0,023 & 0,032 & 0,033 & 0,025 & 0,045 & 0,057 & 0,087 & 0 \\
\hline $\mathrm{A}_{13}$ & 0,045 & 0,057 & 0,1 & 0,075 & 0,091 & 0,069 & 0,043 & 0,143 \\
\hline $\mathrm{A}_{14}$ & 0,068 & 0,113 & 0,1 & 0,075 & 0,091 & 0,092 & 0,087 & 0,143 \\
\hline $\mathrm{A}_{15}$ & 0,091 & 0,064 & 0,067 & 0,1 & 0,045 & 0,069 & 0,043 & 0,143 \\
\hline
\end{tabular}

Após a implementação da formulação matemática do método TODIM, obtém-se o valor global de cada alternativa, apresentados na Tabela 10. Esta tabela apresenta ainda o valor global normalizado e a ordenação de cada alternativa.

Tabela 10 - Valores Finais e Ordenação.

\begin{tabular}{|c|c|c|}
\hline Alternativa & Valor Global Normalizado & Ordenação \\
\hline $\mathrm{A}_{1}$ & 0,6916 & 5 \\
\hline $\mathrm{A}_{2}$ & 0,3862 & 10 \\
\hline $\mathrm{A}_{3}$ & 0,3992 & 9 \\
\hline $\mathrm{A}_{4}$ & 0,6210 & 1 \\
\hline $\mathrm{A}_{5}$ & 1,0000 & 11 \\
\hline $\mathrm{A}_{6}$ & 0,2860 & 15 \\
\hline $\mathrm{A}_{7}$ & 0,0000 & 8 \\
\hline $\mathrm{A}_{8}$ & 0,4407 & 14 \\
\hline $\mathrm{A}_{9}$ & 0,0202 & 12 \\
\hline $\mathrm{A}_{10}$ & 0,2127 & 3 \\
\hline $\mathrm{A}_{11}$ & 0,8576 & 13 \\
\hline $\mathrm{A}_{12}$ & 0,1073 & 4 \\
\hline $\mathrm{A}_{13}$ & 0,7188 & 2 \\
\hline $\mathrm{A}_{14}$ & 0,9372 & 6 \\
\hline $\mathrm{A}_{15}$ & 0,6733 & \\
\hline
\end{tabular}




\subsection{Análise dos Resultados}

Dentre os imóveis avaliados, alguns foram inseridos como referência, apenas para auxiliar a análise, pois já são conhecidos os seus valores de aluguel. Estes imóveis são apresentados na Tabela 11 .

Tabela 11 - Imóveis com o valor do aluguel já definido.

\begin{tabular}{|c|c|}
\hline Imóvel & Valor Mensal do Aluguel \\
\hline $\mathrm{A}_{3}$ & $\mathrm{R} \$ 450,00$ \\
\hline $\mathrm{A}_{4}$ & $\mathrm{R} \$ 650,00$ \\
\hline $\mathrm{A}_{5}$ & $\mathrm{R} \$ 1.500,00$ \\
\hline $\mathrm{A}_{9}$ & $\mathrm{R} \$ 280,00$ \\
\hline $\mathrm{A}_{10}$ & $\mathrm{R} \$ 350,00$ \\
\hline $\mathrm{A}_{11}$ & $\mathrm{R} \$ 930,00$ \\
\hline
\end{tabular}

Desta forma, inserindo estes imóveis com valores conhecidos, pode-se estabelecer uma faixa de valores para os outros imóveis em análise. Por exemplo, neste estudo, verifica-se que $\mathrm{A}_{8}$ está, na ordenação final pelo método TODIM, em $8^{\circ}$ lugar. $\mathrm{A}_{3}$ está em $9^{\circ}$ lugar, tendo como valor de aluguel a quantia de $\mathrm{R} \$ 450,00$, e $\mathrm{A}_{4}$ está em $7^{\circ}$ lugar, devendo ser alugado por $\mathrm{R} \$ 650,00$. Pode-se então dizer que $A_{8}$ deve ser alugado por um valor entre $\mathrm{R} \$ 450,00$ e $\mathrm{R} \$ 650,00$. Da mesma forma, verifica-se que o imóvel $\mathrm{A}_{14}$ está em $2^{\circ}$ lugar. $\mathrm{A}_{5}$ está em $1^{\circ}$ lugar, com valor de aluguel de $\mathrm{R} \$ 1.500,00$, e $\mathrm{A}_{11}$ está em $3^{\circ}$ lugar, com valor de aluguel de $\mathrm{R} \$ 930,00$. Portanto, o imóvel $\mathrm{A}_{14}$ deve ser alugado por um valor entre $\mathrm{R} \$ 1.500,00$ e $\mathrm{R} \$ 930,00$.

Estes intervalos de valores são definidos para cada alternativa em análise, servindo de referência para obter valores de referência para o aluguel. Desta forma, torna-se menos complexo o processo de decisão. A Tabela 12 sugere os intervalos apropriados, para os imóveis com os valores de aluguel não definidos.

Tabela 12 - Valores Sugeridos para Aluguel das Alternativas em Análise.

\begin{tabular}{|c|c|c|}
\hline Imóvel & Ordenação & Valor Mensal do Aluguel \\
\hline $\mathrm{A}_{1}$ & $5^{\circ}$ & Entre $\mathrm{R} \$ 930,00$ e $\mathrm{R} \$ 650,00$ \\
\hline $\mathrm{A}_{2}$ & $10^{\circ}$ & Entre $\mathrm{R} \$ 450,00$ e $\mathrm{R} \$ 350,00$ \\
\hline $\mathrm{A}_{6}$ & $11^{\circ}$ & Entre $\mathrm{R} \$ 450,00$ e $\mathrm{R} \$ 350,00$ \\
\hline $\mathrm{A}_{7}$ & $15^{\circ}$ & Abaixo de $\mathrm{R} \$ 280,00$ \\
\hline $\mathrm{A}_{8}$ & $8^{\circ}$ & Entre $\mathrm{R} \$ 650,00$ e $\mathrm{R} \$ 450,00$ \\
\hline $\mathrm{A}_{12}$ & $13^{\circ}$ & Entre $\mathrm{R} \$ 350,00$ e $\mathrm{R} \$ 280,00$ \\
\hline $\mathrm{A}_{13}$ & $4^{\circ}$ & Entre $\mathrm{R} \$ 930,00$ e $\mathrm{R} \$ 650,00$ \\
\hline $\mathrm{A}_{14}$ & $2^{\circ}$ & Entre $\mathrm{R} \$ 1.500,00$ e $\mathrm{R} \$ 930,00$ \\
\hline $\mathrm{A}_{15}$ & $6^{\circ}$ & Entre $\mathrm{R} \$ 930,00$ e $\mathrm{R} \$ 650,00$ \\
\hline
\end{tabular}

\subsection{Análise de Sensibilidade}

Depois de obtidos os resultados com a implementação do método TODIM, foi realizada a análise de sensibilidade variando o peso do critério de maior importância para os decisores, que é o critério $C_{1}$. Seu peso foi reduzido de cinco para três e não houve variação da 
ordenação das quinze alternativas. Uma outra análise foi realizada variando o valor de $\theta$, índice que representa o fator de atenuação das perdas. Na primeira implementação realizada o valor de utilizado para $\theta$ foi 1 . Na análise de sensibilidade o valor de $\theta$ foi alterado de 1 para 5. Com esta alteração realizada, somente ocorreu alteração na ordenação dos valores de $\mathrm{A}_{13}$ com $\mathrm{A}_{15}$, indicando que, apesar da alteração no valor do índice de atenuação das perdas, a ordenação obtida é consistente.

\section{Conclusão}

A análise das alternativas utilizando o método TODIM conduziu a uma ordenação que se mostrou satisfatória e concordante com as expectativas dos especialistas. Através de sua formulação torna-se mais fácil resolver os conflitos existentes entre os critérios, pois, às vezes, para se conseguir um bom desempenho em um determinado critério de análise, devese abrir mão do desempenho de outro, o que configura uma importante relação de troca (Gomes, 2007).

No caso de avaliação de imóveis, o método é capaz de auxiliar os profissionais do ramo imobiliário a avaliar mais claramente as alternativas em relação aos critérios definidos pelos especialistas.

Neste estudo foi possível identificar ainda que três imóveis, $A_{1}, A_{13}$ e $A_{15}$, caíram dentro da mesma faixa estabelecida pelas alternativas $\mathrm{A}_{4} \mathrm{e} \mathrm{A}_{11}$. Pela Tabela 10 , verifica-se que a ordem desses três imóveis é: $A_{13}, A_{1}$ e $A_{15}$. Desta maneira, os valores de aluguel desses três imóveis poderiam ser os mesmos, ou, de outro modo, referenciando o valor do aluguel do imóvel de acordo com a ordenação fornecida pelo método, isto é, o maior valor para $A_{13}$ e o menor valor para $\mathrm{A}_{15}$.

Assim, a análise e a resolução do problema aqui apresentados, por meio do método multicritério TODIM, refletiu em seus resultados as preferências dos agentes de decisão, conhecedores das múltiplas dimensões do problema analisado. Por conseguinte, pode-se concluir que o método constitui-se em um eficaz auxiliar na avaliação de imóveis.

\section{Agradecimentos}

Os autores agradecem ao Ibmec/RJ, através de seu Programa de Pós-Graduação e Pesquisa em Administração, e ao CNPq, através do Projeto de Pesquisa N.306658/2004-6, por propiciarem meios para a realização desta pesquisa.

\section{Referências Bibliográficas}

(1) Bana e Costa, C.A. \& Vansnick, J.C. (1997). Thoughts on a theoretical framework for measuring attractiveness by categorical based evaluation technique (Macbeth). In: Multicriteria Analysis [edited by J. Clímaco], Springer Verlag.

(2) Barba-Romero, S. \& Pomerol, J.C. (1997). Decisiones multicriterio: fundamentos teóricos y utilización práctica. Servicio de Publicaciones, Universidad de Alcalá, Alcalá.

(3) Belton, V. \& Stewart, T.J. (2002). Multiple criteria decision analysis: an integrated approach. Kluwer Academic Publishers, Massachusetts. 
(4) Brans, J.P. \& Mareschal, B. (1990). The PROMÉTHÉE methods for MCDM, the PROMCALC GAIA and BANDADVISER software. In: Readings in Multiple Criteria Decision Aid [edited by C.A. Bana e Costa], chapter 2, Springer Verlag,

(5) Brans, J.P.; Mareschal, B. \& Vincke, Ph. (1984). PROMÉTHÉE: A new family of outranking methods in multicriteria analysis. In: Operational Research [edited by J.P. Brans], Elsevier Science Publishers B.V., North-Holland, 408-421.

(6) Clemen, R.T. \& Reilly, T. (2001). Making hard decisions with decision tools. Duxbury, Pacific Grove.

(7) Costa, A.P.C.S.; Almeida, A.T. de \& Gomes, L.F.A.M. (2003). Priorização do Portfolio de Projetos de Sistemas de Informação Baseado no Método TODIM de Apoio Multicritério à Decisão. Investigación Operativa, vol.XI, 23, 106-119.

(8) Gomes, L.F.A.M. \& Lima, M.M.P.P. (1992b). From Modeling Individual Preferences to Multicriteria Ranking of Discrete Alternatives: A Look at Prospect Theory and the Additive Difference Model. Foundations of Computing and Decision Sciences, 17(3), 171-184.

(9) Gomes, L.F.A.M.; Araya, M.C.G. \& Carignamo, C. (2004). Tomada de decisão em cenários complexos. Pioneira Thomson Learning, São Paulo.

(10) Gomes, L.F.A.M. \& Duarte, V.C. de A. (1998). Análise Multicritério de Risco: O Método TODIM. Trabalho agraciado com o prêmio de primeiro lugar na categoria de poster no XVII ENEGEP e IV Congresso Internacional de Engenharia Industrial. Universidade Federal Fluminense, Niterói.

(11) Gomes, L.F.A.M.; Duarte, V.C.A. \& Moraes, L. F. R. (1999). Análise Multicritério de Projetos de Produção de Petróleo: Os Métodos PROMÉTHÉE e TODIM. Pesquisa Naval, 12, 251-262.

(12) Gomes, L.F.A.M.; Gomes, C.F.S. \& Almeida, A.T. (2006). Tomada de decisão gerencial: enfoque multicritério. $2^{\mathrm{a}}$ ed., Atlas, São Paulo.

(13) Gomes, L.F.A.M. \& Lima, M.M.P.P. (1992a). TODIM: Basics and Application to Multicriteria Ranking of Projects with Environmental Impacts. Foundations of Computing and Decision Sciences, 16(4), 113-127.

(14) Gomes, L.F.A.M. (1976). A multicriteria decision making framework for the evaluation of forest road investment projects. Thesis of Doctor of Philosophy in Engineering. Institute of Transportation Studies, University of California, Berkeley.

(15) Gomes, L.F.A.M. (2007). Teoria da Decisão. Pioneira Thomson Learning, São Paulo.

(16) Guimarães Neto, C.A. (1992). Critérios de Avaliação e NB502-89. In: Introdução a Engenharia de Avaliações e Perícias Judiciais, Ed. Instituto de Engenharia Legal, São Paulo, 45-60.

(17) Jacquet-Lagrèze, E. \& Siskos, J. (1982). Assessing a set of additive utility functions for multicriteria decision-making, the UTA method. European Journal of Operational Research, 10, 151-164.

(18) Kahneman, D. \& Tversky, A. (1979). Prospect theory: An analysis of decision under risk. Econometrica, 47, 263-292.

(19) Keeney, R.L. \& Raiffa, H. (1993). Decisions with multiple objectives: preferences and value tradeoffs. Cambridge University Press, Cambridge. 
(20) Mourão, Y.B. \& Gomes, L.F.A.M. (2006). Priorização de projetos de pesquisa e desenvolvimento na indústria do petróleo: uma aplicação da teoria dos prospectos. Anais do XXXVIII Simpósio Brasileiro de Pesquisa Operacional. SOBRAPO, Goiânia.

(21) Passos, A.C. \& Gomes, L.F.A.M. (2005). Avaliação Multicritério de Material de Emprego Militar. Revista Militar de Ciência e Tecnologia, 22, 20-29.

(22) Pessôa, M. (1992). Laudos técnicos, conceitos básicos e definições. In: Introdução a Engenharia de Avaliações e Perícias Judiciais, Ed. Instituto de Engenharia Legal, São Paulo, 1-26.

(23) Rangel, L.A.D.; Silva, S.S. da \& Silvério, L.B. (2006). Ordenação de imóveis residenciais empregando o método TODIM. Anais do XXXVIII Simpósio Brasileiro de Pesquisa Operacional. SOBRAPO, Goiânia.

(24) Rangel, L.A.D. (2002). Determinações de funções de utilidade através das preferências dos decisores sobre o conjunto de critérios empregando o método UTA. Tese de Doutorado em Engenharia de Produção. COPPE/ Programa de Engenharia de Produção, Universidade Federal do Rio de Janeiro, Rio de Janeiro.

(25) Roux, D. (2002). Nobel en Économie. $2^{\mathrm{e}}$ édition. Economica, Paris.

(26) Roy, B. \& Bouyssou, D. (1993). Aide multicritère à la décsion: méthodes et cas. Economica, Paris.

(27) Roy, B. \& Hugonnard, J.Ch. (1982). Classement de prolongements de métro en banlieue parisienne, présentation d'une méthode multicritère originale. Cahiers $d u$ CERO, 23, 153-171.

(28) Roy, B. \& Skalka, J.M. (1984). ELECTRE IS: Aspects méthodologiques et guide d'utilisation. Document du Lamsade, 30. Université de Paris-Dauphine, Paris.

(29) Roy, B. (1978). Electre III: Um algorithme de classements fondé sur une représentation floue des préférences en présence de critères multiples. Cahier du CERO, 20(1), 3-24.

(30) Roy, B. \& Bertier, P. (1973). La méthode Electre II - une application au média-planning. In: Operational Research [edited by M. Ross], North-Holland, Amsterdã, 291-302.

(31) Roy, B. (1968). Classement et choix en présence de points de vue multiple: la méthode Electre. R.I.R.O., 8, 57-75.

(32) Saaty, T.L. (1990). Decision making for leaders: the analytic hierarchy process for decisions in a complex world. RWS Publications, Pittsburgh.

(33) Salminen, P. (1992). Descriptive and Prescriptive Modelling of Preferences within a Discrete Alternative Multiple Criteria Decision Framework. Jyväskylä Studies in Computer Science, Economics and Statistics, 19. University of Jyväskylä, Jyväskylä.

(34) Trotta, L.T.F.; Nobre, F.F. \& Gomes, L.F.A.M. (1999). Multi-Criteria Decision Making - An Approach to Setting Priorities in Health Care. Statistics in Medicine, 18, 3345-3354. 